\title{
Architecture for the Performance of Nuclear Fuel Depletion Calculations
}

Olin William Calvin, Mark D DeHart

November 2019

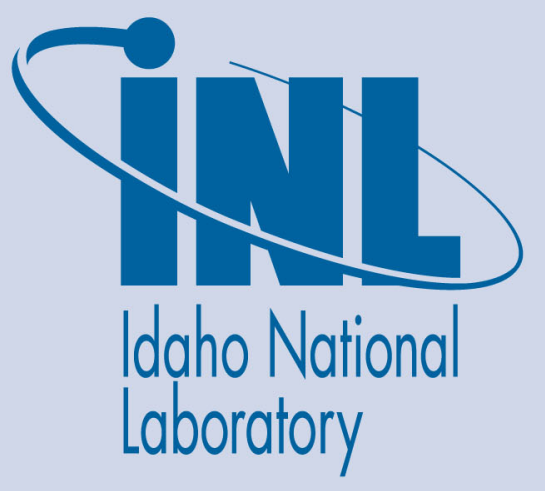

The INL is a U.S. Department of Energy National Laboratory operated by Battelle Energy Alliance 


\title{
Architecture for the Performance of Nuclear Fuel Depletion Calculations
}

\author{
Olin William Calvin, Mark D DeHart
}

November 2019

Idaho National Laboratory Idaho Falls, Idaho 83415

http://www.inl.gov

Prepared for the

U.S. Department of Energy

Under DOE Idaho Operations Office

Contract DE-AC07-05ID14517 


\title{
Architecture for the Performance of Nuclear Fuel Depletion Calculations
}

\author{
Olin William Calvin \\ Dr. Mark DeHart, PhD \\ University of Florida \\ Herbert Wertheim College of Engineering \\ Department of Materials Science and Engineering \\ Nuclear Engineering Program
}

October 2019 


\section{Abstract}

Computational analysis of the depletion of nuclear fuel is crucial to the accurate modeling of the time-dependent behavior of nuclear reactors. The reactor physics application MAMMOTH is modified to compute radioactive decay and neutron transmutation in order to model physical processes which occur within nuclear reactors as reactor fuel is burned. The ability to read decay and cross-section data files of other codes are also added and the addition of a standardized decay and transmutation XML file format for MAMMOTH, known as ISOXML. Comparisons between MAMMOTH and the Oak Ridge Isotope Generation (ORIGEN) code are performed to demonstrate the improved precision possible with MAMMOTH. In addition, several tests are run to demonstrate the ability of MAMMOTH to model decay and transmutation processes involving hundreds of isotopes. Future work will focus on solving depletion problems with multiple neutron energy groups and the implementation of predictor-corrector methods, several of which are described in this work.

\section{Introduction}

Nuclear engineering, at its core, involves the study and analysis of nuclear processes, such as radioactive decay and radiation-induced transmutation, typically as a result of neutron-nuclei interactions. Transmutation in the form of nuclear fission is what allows nuclear reactors to release heat, which can subsequently be used to generate electricity. These fission events both rely on radioactive elements, such as uranium and plutonium, while also subsequently producing numerous radioactive isotopes which undergo subsequent radioactive decay. The time scales involved in these events present computational challenges as the half-lives of these isotopes can vary wildly, with uranium-238 having a half-life of $4.5 * 10^{9}$ years while the short-lived isotope helium-5 has a half-life of $7.0 * 10^{-22}$ seconds. Half-lives, and subsequently decay constants, that vary by over forty orders of magnitude can introduce serious challenges when attempting to numerically solve the system of equations to account for the relative compositions of each nuclei for a given nuclear system.

Idaho National Laboratory has developed the MAMMOTH reactor physics application [1] for the analysis of numerous reactor experiments. MAMMOTH is based on the Multiphysics Object-Oriented Simulation Environment (MOOSE) framework [2], [3], upon which numerous other applications have been built, such as the fuel performance application Bison [4] and the thermal fluids application RELAP-7 [5].

This work documents the implementation of microscopic depletion functionality into MAMMOTH as well as the ability to read ORIGEN-formatted ENDF decay data files for use within MAMMOTH. This data allows MAMMOTH to solve nuclear transmutation problems with the same data set as ORIGEN (over 1,600 isotopes) [6] allowing a direct comparison of numerical results between the two applications independent of the data. Three tests are performed in order to ensure the efficacy of the results produced by the newly implemented functionality. 


\section{Background}

The equations to describe the behavior of radioactive decay and neutron reactions were developed over the course of the early and mid twentieth century. With the development of commercial nuclear reactors and the importance of radioactive decay and neutron cross sections, numerous computational methods for calculating and modeling the depletion of nuclear fuel have been developed in an effort to solve or approximate these equations.

\subsection{The Bateman Equation}

The mathematical model for determining the time-dependent abundance and rate of decay for a given radioactive nuclide can be described through the Bateman equation. This analytical solution was developed by Harry Bateman in 1910 [7]. The simplest form of a series of Bateman equations is for the decay of a single radioactive isotope with decay constant $\lambda$ to a stable isotope. An example, the beta decay of carbon-14 to stable nitrogen-14, is shown in Equation 1:

$$
\frac{d N_{C 14}(t)}{d t}=-\lambda_{C 14} N_{C 14}(t)
$$

where

$\frac{d N_{C 14}(t)}{d t}$ is the time-dependent rate of change in the number density of carbon-14

$\lambda_{C 14}$ is the decay constant of carbon-14

$N_{C 14}(t)$ is the time-dependent number density of carbon-14

The Bateman equation can be further expanded to account for the processes of nuclear transmutation. The inclusion of neutron transmutation terms to the Bateman equation is demonstrated by Bell and Glasstone [8]. Expanding upon the carbon-14 example, if carbon-14 is exposed to a constant monoenergetic neutron flux and it is asserted that carbon-14 can undergo a radiative neutron capture $(\mathrm{n}, \gamma)$ reaction to become carbon-15 and that carbon-13 can undergo a radiative neutron capture reaction to become carbon14, the equation becomes Equation 2, where:

$$
\frac{d N_{C 14}(t)}{d t}=\sigma_{\gamma, C 13} N_{C 13}(t) \phi-\sigma_{\gamma, C 14} N_{C 14}(t) \phi-\lambda_{C 14} N_{C 14}(t)
$$

where

$\frac{d N_{C 14}(t)}{d t}$ is the time-dependent rate of change in the number density of carbon-14

$\sigma_{\gamma, C 13}$ is the microscopic radiative capture cross section of carbon-13

$N_{C 13}(t)$ is the time-dependent number of carbon-13 atoms

$\phi$ is the monoenergetic neutron flux within the system

$\sigma_{\gamma, C 14}$ is the microscopic radiative capture cross section of carbon-14

$N_{C 14}(t)$ is the time-dependent number of carbon-14 atoms

$\lambda_{C 14}$ is the decay constant of carbon-14

More generically, the time-dependent rate of change in number density for any nuclide $i$ can be defined in Equation 3 as a modified version of an equation shown in Bell and Glasstone [8]: 


$$
\begin{array}{r}
\frac{d N_{i}(t)}{d t}=\sum_{j} \gamma_{j, i} \sigma_{f, j} N_{j}(t) \phi+\sum_{k}\left(\sum_{r} \sigma_{r, k} N_{k}(t) \phi\right)+\sum_{l} \lambda_{l} N_{l}(t) \\
-\sum_{m} \sigma_{m, i} N_{i}(t) \phi-\lambda_{i} N_{i}(t)
\end{array}
$$

where

$\frac{d N_{i}(t)}{d t}$ the time-dependent rate of change in the number density of nuclide $i$

$\gamma_{j, i}$ the probability that nuclide $i$ will be produced as a fission product as a result of the neutron-induced nuclear fission of nuclide $j$

$\sigma_{f, j}$ the microscopic fission cross section of nuclide $j$

$N_{j}(t)$ the time-dependent number density of nuclide $j$

$\phi$ the monoenergetic neutron flux within the system

$\sigma_{r, k}$ the microscopic non-scattering, non-fission reaction cross section of reaction type $r$ of nuclide $k$ which produces nuclide $i$

$N_{k}(t)$ the time-dependent number density of nuclide $k$ which produces nuclide $i$ as a result of reaction $r$

$\lambda_{l}$ the decay constant of nuclide $l$ which produces nuclide $i$ as a result of radioactive decay

$N_{l}(t)$ the time-dependent number density of nuclide $l$

$N_{i}(t)$ the time-dependent number density of nuclide $i$

$\sigma_{m, i}$ the microscopic non-scattering, reaction cross section of reaction type $m$ of nuclide $i$

$\lambda_{i}$ the decay constant of nuclide $i$

\subsection{The Depletion Matrix}

Since for a set of $I$ nuclides of interest the Bateman equations form a system of firstorder linear differential equations, the system can be written in matrix form as shown in Equation 4.

$$
\frac{d \mathbf{n}}{d t}=\mathbf{A n}(t)
$$

where

$\mathbf{n}(t) \in \mathbb{R}^{n}$ is the time-dependent nuclide number density vector of rank $I$

$\mathbf{A} \in \mathbb{R}^{n \times n}$ is the depletion matrix with the decay and transmutation coefficients for each nuclide in the system

Matrix A, as shown in Equation 4, depends on the decay constants, cross sections, and neutron flux within the depletion system. Cross sections may or may not be timedependent, depending on the data available, and the neutron flux level may or may not be time-dependent. In constant power depletion the neutron flux level must change in order to account for changing reaction rates in the depletion system while for constant flux depletion the flux is time-independent. Thus matrix A may or may not be time-dependent, this is significant as analytical solutions for systems where $\mathbf{A}$ is time-independent are 
significantly easier to calculate, even for systems with hundreds of nuclides, than for time-dependent systems.

As a result of the dependence of matrix $\mathbf{A}$ on decay constants, cross sections, and neutron flux, the matrix can be further decomposed into the form shown in Equation 5.

$$
\mathbf{A}=\mathbf{A}_{\mathrm{DC}}+\mathbf{A}_{\mathrm{XS}} \phi
$$

where

A is the depletion matrix accounting for both radioactive decay and neutron transmutation

$\mathbf{A}_{\mathrm{DC}}$ is the matrix accounting for the radioactive decay constants

$\mathbf{A}_{\mathrm{XS}}$ is the matrix accounting for the neutron reaction cross sections

$\phi$ is the neutron flux in the system

An advantage of decomposing the depletion matrix in this way is the ability to isolate any time dependence in the matrix. Since decay constants are always time-independent, the decay part of the depletion matrix, referred to as the decay matrix, only needs to be populated with the necessary decay data once for a given problem. The cross section matrix and neutron flux can be time-independent depending on the problem statement, however most real-world reactor problems require both cross sections and neutron flux to be time-dependent meaning that this portion of the matrix must be recalculated throughout the problem as the neutron flux and cross sections change over time.

An example of the formation of the depletion matrix $\mathbf{A}$ can be shown using the carbon-14 example in Equation 2 if the equation for the number density of carbon-13 is also added as shown in Equation 6 to account for the loss of carbon-13 as it transmutes by radiative neutron capture $(n, \gamma)$ into carbon- 14 .

$$
\frac{d N_{C 13}(t)}{d t}=-\sigma_{\gamma, C 13} N_{C 13}(t) \phi
$$

The combination of Equations 2 and 6 results in the equation shown in Equation 7 where:

$$
\left[\begin{array}{c}
\frac{d N_{C 14}(t)}{d t} \\
\frac{d N_{C 13}(t)}{d t}
\end{array}\right]=\left[\begin{array}{cc}
-\lambda_{C 14}-\sigma_{\gamma, C 14} \phi & \sigma_{\gamma, C 13} \phi \\
0 & -\sigma_{\gamma, C 13} \phi
\end{array}\right]\left[\begin{array}{c}
N_{C 14}(t) \\
N_{C 13}(t)
\end{array}\right]
$$

Using the decomposition in Equation 5, Equation 7 can also be rewritten as shown in Equation 8:

$$
\left[\begin{array}{l}
\frac{d N_{C 14}(t)}{d t} \\
\frac{d N_{C 13}(t)}{d t}
\end{array}\right]=\left\{\left[\begin{array}{cc}
-\lambda_{C 14} & 0 \\
0 & 0
\end{array}\right]+\left[\begin{array}{cc}
-\sigma_{\gamma, C 14} \phi & \sigma_{\gamma, C 13} \phi \\
0 & -\sigma_{\gamma, C 13} \phi
\end{array}\right]\right\}\left[\begin{array}{l}
N_{C 14}(t) \\
N_{C 13}(t)
\end{array}\right]
$$

If we go back to Equation 4 and assert that $\mathbf{n}(0)=\mathbf{n}_{0}$ then the Bateman equations can be treated as a single matrix differential equation [9] as shown in Equation 9:

$$
\mathbf{n}(t)=e^{\mathbf{A} t} \mathbf{n}_{0}
$$

The exponential of the matrix $\mathbf{A} t$ is defined as the power series expression [10] in Equation 10: 


$$
e^{\mathbf{A} t}=\sum_{k=0}^{\infty} \frac{1}{k !}(\mathbf{A} t)^{k}
$$

Because of the the magnitude of the variation in decay constants between various nuclides, as well as the potential for substantial variation in microscopic cross sections of materials, many methods for solving the matrix exponential are either not suitable for use in depletion calculations or must be heavily modified to account for the orders of magnitude differences that can occur between coefficients.

\subsection{Chebyshev Rational Approximation Method (CRAM)}

The Chebyshev Rational Approximation Method (CRAM) was first applied to depletion/burnup calculations within the last decade [11] and has become widely used with several depletion-capable codes, such as Serpent 2 and more recently as an option in ORIGEN, to solve the Bateman equation system. A very brief overview of the method is provided here.

The Chebyshev Rational Approximation Method relies on a rational function $r(z)$ in order to best approximate the exponential function developed in the Bateman equations in the complex plane. CRAM was originally derived based on the Cauchy integral formula allowing the matrix exponential to be written in the form of Equation 11:

$$
e^{\mathbf{A} t}=\frac{1}{2 \pi i} \int_{\Gamma} e^{z}(z \mathbf{I}-\mathbf{A} t)^{-1} d z
$$

where

$\Gamma$ is a closed contour winding once around the spectrum of $\mathbf{A} t$

Pusa [11] demonstrated that Equation 11 could be used to simplify calculation of the matrix exponential into the calculation of contour integrals and, since depletion ultimately involves real numbers only, the solutions to the system of equations is confined to poles on the negative real axis allowing the calculation of the number densities in a depletion system to reduce to the form shown in Equation 12:

$$
\mathbf{n}=\alpha_{0} \mathbf{n}_{0}+2 \operatorname{Re}\left(\sum_{j=1}^{k / 2} \alpha_{j}\left(\mathbf{A} t-\theta_{j} \mathbf{I}\right)^{-1} \mathbf{n}_{0}\right)
$$

where

$k$ is the order of the CRAM solver

$\alpha \& \theta$ are constants given by documentation on CRAM

$t$ is the length of the time step

$\mathbf{n}$ is the vector of nuclide number densities at time $t$

$\mathbf{n}_{0}$ is the vector of the initial nuclide number densities before the depletion time step

A is the depletion matrix

$\mathbf{I}$ is the identity matrix of same size as $\mathbf{A}$

Re is the real portion of the calculated summation 


\subsection{Mini-Max Polynomial Approximation Method}

The Mini-Max Polynomial Approximation (MMPA) method was developed by Yosuke Kawamoto [12] as an alternative to CRAM for solving the Bateman equations. While this method is not as widely adopted as CRAM, it was also implemented in MAMMOTH to serve as a way to verify the results of CRAM. A brief overview of the method is provided here.

The MMPA method reduces solving the matrix exponential $e^{\mathbf{A} t}$ by approximating it as a function $f$ of a given order by first defining $f$ as a polynomial function as shown in Equation 13:

$$
f(t)=\alpha_{0}+\sum_{i=1}^{n} \alpha_{i} t^{i}
$$

where

$$
\begin{aligned}
\alpha_{i} \in \mathbb{R} & \text { are approximation coefficients } \\
n & \text { is an expansion order }
\end{aligned}
$$

Asserting a given exponential function of the form:

$$
e^{c \frac{t+1}{t-1}}
$$

where

$$
c \in \mathbb{R} \text { is an arbitrary positive constant }
$$

$(t+1) /(t-1)$ restricts $t$ to $[-1,1)$. The coefficients $\alpha_{i}$ in Equation 13 are defined to minimize the error between Equations 13 and 14:

$$
\left|f(t)-e^{c \frac{t+1}{t-1}}\right|
$$

The variable $t$ is substituted as follows:

$$
t=\frac{x+c}{x-c}
$$

This substitution allows the approximation equation to take the form of Equation 17:

$$
e^{x} \approx f(x)=\alpha_{0}+\sum_{i=1}^{n} \alpha_{i}\left(\frac{x+c}{x-c}\right)^{i}
$$

where

$$
x \in(-\infty, 0]
$$

With the form developed in Equation 17, the system can be modified to accommodate matrix form by substituting $x$ with $\mathbf{A} t$ and $c$ with $c \mathbf{I}$ where $\mathbf{I}$ is the identity matrix of the same size as matrix A, resulting in the polynomial approximation shown in Equation 18:

$$
\mathbf{n}(t)=\alpha_{0} \mathbf{I n}_{0}+\sum_{i=1}^{k} \alpha_{i}\left\{(\mathbf{A} t+c \mathbf{I})(\mathbf{A} t-c \mathbf{I})^{-1} \mathbf{n}_{0}\right\}
$$


where
$k$ is the order of the CRAM solver
$\alpha \& c$ are constants given by documentation on MMPA
$t$ is the length of the time step
$\mathbf{n}$ is the vector of nuclide number densities at time $t$
$\mathbf{n}_{0}$ is the vector of the initial nuclide number densities before the depletion time step
A is the depletion matrix
$\mathbf{I}$ is the identity matrix of same size as $\mathbf{A}$

\subsection{Predictor-Corrector Methods}

Because of the computational costs typically associated with high fidelity neutron transport calculations, depletion codes separate depletion and neutron transport calculations into two distinct steps, rather than attempt to perform a "persistent" calculation where new neutron fluxes and cross sections are calculated as each individual nuclide in the system changes. A neutron transport calculation is performed in order to determine the neutron flux in the system as well as other multiphysics variables such as temperature, which cross sections depend on. The neutron flux and cross sections are used to calculate the neutron transmutation reaction rates for each isotope present in the system. When combined with the known decay rates for each isotope, this allows the system to be depleted over a given time period using said reaction rates. Thus, the new number densities for each isotope in the system can be determined. However, since the number density of each isotope changes over time, the neutronics of the system change.

In a light water nuclear reactor, which typically operates at a constant power level, this requires the neutron flux within a reactor to increase over time to account for the buildup of neutron poisons, such as xenon-135, and the net loss of fissile material within the reactor over time, such as uranium-235. As a result, the reaction rates of the system change during the depletion time steps, however the depletion matrix cannot be updated to account for these neutronic changes while depleting the material because of the separation between neutron transport calculation and the depletion calculation. As a result, numerous methods have been developed in order to predict and/or correct for these changes in an effort to improve accuracy while decreasing the computational costs of depletion calculations. Methods used by the codes Serpent 2 and TRITON (Transport Rigor Implemented with Time-dependent Operation for Neutronic depletion) are examined as well as other published predictor-corrector methods.

Some of these predictor-corrector methods are:

- Constant Extrapolation (CE), available in Serpent 1 \& 2 [13]

- Constant Extrapolation with Linear Interpolation (CE/LI), available in Serpent 1 \& $2[13]$

- Linear Extrapolation (LE), available in Serpent 2 [13]

- Linear Extrapolation with Linear Interpolation (LE/LI), available in Serpent 2 [13]

- Linear Extrapolation with Quadratic Interpolation (LE/QI), available in Serpent 2 [13]

- TRITON Method [14] 
- MONTEBURNS [15]

- CELL-2 [16]

\subsubsection{Constant Extrapolation}

The Constant Extrapolation method is the simplest form of predictor-corrector in that it does essentially no prediction or correction over its given time step. The steps for CE are:

1. Perform a neutron transport calculation to determine the neutron fluxes and cross sections at the beginning of the time step.

2. Deplete over the time step using the reaction rates determined by the fluxes and cross sections calculated in Step 1 to solve the Bateman equations and determine the number densities at the end of the time step.

3. Begin the next time step using the number densities calculated in Step 2.

The main advantage of Constant Extrapolation is its simplicity and the need to only perform a single neutron transport calculation for each time step. The accuracy of CE can be improved by decreasing the length of the time step, however this consequently results in an increase in computational time and is generally not a preferred alternative when other methods are available. As a result, this method is considered the least accurate predictor/corrector method.

\subsubsection{Constant Extrapolation with Linear Interpolation}

In order to improve accuracy, the Constant Extrapolation with Linear Interpolation method interpolates between the beginning of the time step and the end of the time step in an attempt to account for changes within the system during the depletion time step. The steps for CE/LI are:

1. Perform a neutron transport calculation to determine the neutron fluxes and cross sections at the beginning of the time step.

2. Deplete over the time step using the reaction rates determined by the fluxes and cross sections calculated in Step 1 to solve the Bateman equations and determine the number densities at the end of the time step.

3. Perform a neutron transport calculation to determine the neutron fluxes and cross sections at the end of the time step using the number densities calculated in Step 2 .

4. Deplete over the entire time step using the initial number densities and the average of the beginning and end of time step neutron fluxes and cross sections, calculated in Steps 1 and 3 respectively, to solve the Bateman equations and determine the final number densities.

5. Begin the next time step using the number densities calculated in Step 4. 
The drawback of CE/LI is the need to perform two neutron transport calculations for each time step, effectively doubling its computational time compared to Constant Extrapolation. However, it is generally considered more accurate than CE with twice as many time steps, since the addition of interpolation allows the algorithm to better correct for the change in number densities for each given isotope over each time step.

\subsubsection{Linear Extrapolation}

Linear Extrapolation relies on the usage of information from the previous step to extrapolate the behavior of the current time step after calculating the flux and cross sections at the beginning of the time step. The steps for LE are:

1. Perform a neutron transport calculation to determine the neutron fluxes and cross sections at the beginning of the time step.

2. Use the neutron fluxes and cross sections from the beginning of the previous time step and the fluxes and cross sections calculated in Step 1 to linearly extrapolate the anticipated fluxes and cross sections at the end of the current time step.

3. Deplete over the time step using the reaction rates determined by the average of the beginning and extrapolated fluxes and cross sections, calculated in Steps 1 and 2 respectively, to solve the Bateman equations and determine the number densities at the end of the time step.

4. Begin the next time step using the number densities calculated in Step 3 and save the fluxes and cross sections calculated in Step 1 for use in the extrapolation of the next time step.

Linear Extrapolation improves upon Constant Extrapolation while only requiring one neutron transport calculation. LE is typically more accurate than $\mathrm{CE}$ for the same length of time steps. The only detriment to the Linear Extrapolation method is the increased memory requirements due to the need to store the reaction rates and number densities from the previous time step. Since it is impossible to extrapolate during the first time step (since there is no previous time step to extrapolate from), the first time step is depleted using CE. This can be problematic as cross sections are strongly affected by the buildup of poisons such xenon-135 and samarium-149 early in the life of the core. This can be addressed by deliberately performing very short time steps during reactor startup before transitioning to longer time steps after poisons have reached saturation concentration.

\subsubsection{Linear Extrapolation with Linear Interpolation}

Linear Interpolation with Linear Extrapolation combines two of the methods above and requires two neutron transport solves. The steps for LE/LI are:

1. Perform a neutron transport calculation to determine the neutron fluxes and cross sections at the beginning of the time step.

2. Use the neutron fluxes and cross sections from the beginning of the previous time step and the fluxes and cross sections calculated in Step 1 to linearly extrapolate the anticipated fluxes and cross sections at the end of the current time step. 
3. Deplete over the time step using the reaction rates determined by the average of the beginning and extrapolated fluxes and cross sections, calculated in Steps 1 and 2 respectively, to solve the Bateman equations and determine the number densities at the end of the time step.

4. Perform a neutron transport calculation to determine the neutron fluxes and cross sections at the end of the time step based on the number densities calculated in Step 3.

5. Deplete over the entire time step using the initial number densities and the average of the beginning and end of time step neutron fluxes and cross sections, calculated in Steps 1 and 4 respectively, to solve the Bateman equations and determine the number densities at the end of the time step.

6. Begin the next time step using the number densities calculated in Step 3 and save the fluxes and cross sections calculated in Step 5 for use in the extrapolation of the next time step.

This method requires two neutron transport calculations per time step. It is regarded as an improvement on both Linear Extrapolation and Constant Extrapolation with Linear Interpolation, however it does require more memory than $\mathrm{CE} / \mathrm{LI}$ and it does require double the neutron transport calculations as LE for the same number of time steps. Since it is impossible to extrapolate during the first time step (since there is no previous time step to extrapolate from), the first time step is depleted using CE/LI. This introduces the same issues during the first few depletion time steps as in the LE approach.

\subsubsection{Linear Extrapolation and Quadratic Interpolation}

Linear Extrapolation with Quadratic Interpolation incorporates the values from the previous step into the interpolation process through a second-order polynomial. The steps of LE/QI are:

1. Perform a neutron transport calculation to determine the neutron fluxes and cross sections at the beginning of the time step.

2. Use the neutron fluxes and cross section from the beginning of the previous time step and the fluxes and cross sections calculated in Step 1 to linearly extrapolate the anticipated fluxes and cross sections at the end of the current time step.

3. Deplete over the time step using the reaction rates determined by the average of the beginning and extrapolated fluxes and cross sections, calculated in Steps 1 and 2 respectively, to solve the Bateman equations and determine the number densities at the end of the time step.

4. Perform a neutron transport calculation to determine the neutron fluxes and cross sections at the end of the time step based on the number densities calculated in Step 3.

5. Deplete over the entire time step using the initial number densities and the quadratic interpolation of the neutron fluxes and cross sections from the beginning of the previous time step, the beginning of the current time step (calculated in Step 1), and the end of the current time step (calculated in Step 4), to solve the Bateman equations and determine the number densities at the end of the time step. 
6. Begin the next time step using the number densities calculated in Step 5 and save the fluxes and cross sections calculated in Step 1 for use in the extrapolation and interpolation for the next time step.

This method requires two neutron transport calculations per time step and, because of the need to store the fluxes and cross sections of the previous step for any extrapolation algorithm, LE/QI is no more computationally expensive than LE/LI. Since it is impossible to quadratically interpolate or linearly extrapolate during the initial time step (since there is no previous time step to extrapolate or interpolate from), the first time step is depleted using CE/LI. Once again this introduces the same problem observed in the LE and LE/LI approaches.

\subsubsection{TRITON Method}

The predictor-corrector method used by TRITON is not given a specific name and is thus referred to here as the TRITON method, the origins of which come from the depletion approach used in the 1-D depletion sequence SAS2H [17]. The steps of the TRITON method are:

1. Deplete over half of the time step using the reaction rates determined by the fluxes and cross sections from the midpoint of the previous time step.

2. Perform a neutron transport calculation to determine the neutron fluxes and cross sections at the midpoint of the current time step using the number densities calculated in Step 1.

3. Deplete over the entire time step using the initial number densities and the reaction rates determined by the fluxes and cross sections calculated in Step 2.

4. Begin the next time step using the number densities calculated in Step 3 and save the fluxes and cross sections calculated in Step 2 for use in the initial half-step depletion.

This method requires one neutron transport calculation per time step except for the initial time step. Since there is no previous midpoint during the initial time step, a neutron transport calculation is performed at the beginning of the initial time step in order to determine the reaction rates necessary for the initial depletion calculation.

\subsubsection{MONTEBURNS}

The MONTEBURNS method involves the following steps:

1. Perform a neutron transport calculation to determine the neutron fluxes and cross sections at the beginning of the time step.

2. Deplete over half of the time step using the reaction rates determined by the fluxes and cross sections calculated in Step 1.

3. Perform a neutron transport calculation at the midpoint of the time step to recalculate the neutron fluxes and cross sections. 
4. Deplete over the entire time step using the reaction rates determined by the fluxes and cross sections calculated in Step 3.

5. Begin the next time step using the number densities calculated in Step 4.

The MONTEBURNS method requires two neutron transport calculations per time step.

\subsubsection{CELL2}

The CELL2 method involves the following steps:

1. Perform a neutron transport calculation to determine the neutron fluxes and cross sections at the beginning of the time step.

2. Deplete over the time step using the reaction rates determined by the fluxes and cross sections calculated in Step 1.

3. Perform a neutron transport calculation at the end of the time step to calculate the neutron fluxes and cross sections.

4. Deplete over the entire time step using the reaction rates determined by the fluxes and cross sections calculated in Step 3.

5. Begin the next time step using the average of the number densities calculated in Steps 2 and 4.

The CELL2 method requires two transport calculations per time step.

\subsubsection{MAMMOTH Implementation}

MAMMOTH utilizes the CELL-2 method because of its simplicity and consistency when applied to a variety of problems. Other predictor-corrector methods may be implemented in the future, but presently the CELL-2 method provides satisfactory results without increasing memory requirements when compared to other predictor-corrector methods, which could become a constraint as MAMMOTH undergoes continued development. Additionally, CELL-2 does not suffer from the initial time step exceptions which are experienced by the LE, LE/LI, LE/QI, and TRITON methods.

\section{$3 \quad$ ISOXML Library Development}

MAMMOTH is a general reactor physics application built using the Multiphysics Object-Oriented Simulation Environment (MOOSE) framework developed and maintained by Idaho National Laboratory. Considerable effort was spent implementing new depletion capabilities to the MAMMOTH application. As a result, MAMMOTH is now capable of performing radioactive decay and neutron transmutation under monoenergetic, one-group flux conditions. Three benchmark problems are solved in order to verify the accuracy and correctness of the depletion implementation in MAMMOTH. 


\subsection{Decay Data Loader}

In order to perform depletion problems, the radioactive decay data for all the nuclides being tracked in the system must be known. ORIGEN utilizes one of the most extensive decay data libraries assembled, based on the ENDF data set, in order to track the radioactive decay properties of over 1,600 nuclides. A reader was implemented into MAMMOTH in order to read the ORIGEN data files for both the radioactive decay data and the fission product yield fractions for neutron-induced fission events distributed in SCALE 6.2. In addition, cross-section data loaders were implemented to read the binary one-group cross-section data files distributed with ORIGEN in SCALE 6.1 which contain cross sections for over 1,400 nuclides. These data files subsequently underwent a format change from SCALE 6.1 to 6.2 , the documentation of this format not being publicly available in the SCALE user manual.

\subsection{The ISOXML Format}

An XML-based format, known as ISOXML, was developed in order to store the decay data and fission product yield fraction data used by MAMMOTH. The following subsections describe this new data format.

\subsubsection{Terminology}

For clarity, several terms are defined which will be used frequently.

1. Decay reaction: Type of reactions due to radioactive decay. Valid decay reactions are listed in Table 1.

2. Transmutation reaction: Type of reactions initiated from neutron capture. Valid transmutation reactions are listed in Table 2.

3. ZAID: Unique identifier given to each nuclide in the form of ZZAAAM where ZZ is the Z-number of the nuclide (nuclides with $\mathrm{Z}>99$ are not permitted), AAA is the A-number or mass number of the nuclide, and $\mathrm{M}$ is the nuclear isomeric state of the nuclide (0 for ground state nuclides). This identification system was introduced in ENDF-VI. Examples: hydrogen-1 has a ZAID of 10010 and the nuclear isomer of sodium-24 has a ZAID of 110241.

\subsubsection{Format}

The XML format of the decay and transmutation library is defined in this section. The name (XML tag) of the root element is always DecayTransmutationLibrary and its attributes are:

- Name

Description: the name of the library

Data type: string

Default value: empty string

Selection: any valid string 
Table 1: Valid decay reaction types

\begin{tabular}{|c|c|c|}
\hline Identifier & Reaction & Description \\
\hline GenericDecay & Decay & generic decay (could be a chain of decays) \\
Betam & $\beta^{-}$ & electron production \\
Betap & $\beta^{+}$ & positron production or electron capture \\
DBetam & $2 \beta^{-}$ & double electron production \\
Alpha & $\alpha$ & alpha production \\
BetamAlpha & $\beta^{-}$ & electron and alpha production \\
IsomericTransition & $I T$ & isomeric transition \\
SpontaneousFission & $S F$ & spontaneous fission \\
Neutron & $\mathrm{N}$ & neutron production \\
DelayedNeutron & $\beta^{-} \mathrm{N}$ & electron and neutron production \\
\hline
\end{tabular}

Table 2: Valid neutron reaction types (consistent with the reaction types in the MAMMOTH ISOXML multigroup library).

\begin{tabular}{|c|c|c|}
\hline Identifier & Reaction & Description \\
\hline NGamma & $(n, \gamma)$ & radiative capture \\
NAlpha & $(n, \alpha)$ & alpha production \\
N2Alpha & $(n, 2 \alpha)$ & 2 alpha production \\
N2N & $(n, 2 n)$ & 2 neutron production \\
N3N & $(n, 3 n)$ & 3 neutron production \\
N4N & $(n, 4 n)$ & 4 neutron production \\
NProton & $(n, p)$ & proton production \\
NNProton & $(n, n+p)$ & proton + neutron production \\
N2NProton & $(n, 2 n+p)$ & proton +2 neutron production \\
NDeuteron & $(n, d)$ & deuteron production \\
NTriton & $(n, t)$ & triton production \\
Fission & $(n, f)$ & fission \\
\hline
\end{tabular}


- $V e r$

Description: the version of the decay library format

Data type: string

Default value: empty string

Selection: 1.0 is the only valid value at this time; serving as a placeholder for future development.

- Generator

Description: the name of the generator of the library

Data type: string

Default value: "INL"

Selection: any valid string.

The name (XML tag) of the subsequent element(s) is Isotope, which defines an isotope in the library. Its attributes are:

- Name

Description: the name of the isotope

Data type: string

Default value: empty string

Selection: any valid string

- $Z A I D$

Description: the ZAID number for this isotope based on ENDF-VI.

Data type: unsigned integer

Default value: 0

- DecayConstant

Description: the decay constant for this isotope in units of $\left[s^{-1}\right]$

Data type: real

Default value: 0.0

The valid XML tags under the Isotope tag include:

- DecayTypes

Description: list of non-fission decay reactions for this isotope

Data type: DecayType enum vector

Default value: empty vector

- DecayEnergies

Description: Recoverable energy for each non-fission decay reaction for this isotope in units of $[\mathrm{MeV}]$

Data type: real vector

Default value: empty vector 
- DecayGammaEnergyFractions

Description: Fraction of recoverable decay energy released in the form of gamma rays for the decay reaction specified in DecayTypes

Data type: real vector

Default value: empty vector

- DecayBranchingRatios

Description: List of non-fission decay reaction branching ratios for this isotope

Data type: real vector

Default value: empty vector

- DecayDaughters

Description: list of non-fission decay daughters generated by this isotope

Data type: string vector

Default value: empty vector

- NXTypes

Description: list of non-fission $(\mathrm{n}, \mathrm{x})$ reactions for this isotope

Data type: ReactionType enum vector

Default value: empty vector

- NXEnergies

Description: Recoverable energy for each non-fission $(\mathrm{n}, \mathrm{x})$ reaction for this isotope in units of $[\mathrm{MeV}]$

Data type: real vector

Default value: empty vector

- NXGammaEnergyFractions

Description: Fraction of recoverable non-fission $(\mathrm{n}, \mathrm{x})$ energy released in the form of gamma rays for the $(\mathrm{n}, \mathrm{x})$ reaction specified in NXTypes

Data type: real vector

Default value: empty vector

- NXBranchingRatios

Description: List of non-fission $(\mathrm{n}, \mathrm{x})$ reaction branching ratios for this isotope

Data type: real vector

Default value: empty vector

- NXDaughters

Description: list of non-fission $(\mathrm{n}, \mathrm{x})$ daughters generated by this isotope

Data type: string vector

Default value: empty vector 
- FissionData

Description: contain the fission data from neutron-induced and/or spontaneous fission data events and is only present if the isotope can undergo either type of fission.

The valid XML tags under the FissionData tag include:

- NeutronInduced

Description: contain the data from neutron induced reactions.

- Spontaneous

Description: describe the data from spontaneous fission events.

The valid XML tags under the NeutronInduced tag include:

- IncidentNeutronEnergy

Description: list neutron energies in units of $[\mathrm{MeV}]$ for the neutron induced fission reaction tabulations. A value of 0 indicates that the isotope is capable of undergoing neutron-induced fission, but the incident neutron energy and subsequent fission product yields are not known.

Data type: real vector

Default value: empty vector

- FissionTable

Description: contains the data tables from neutron induced fission events for an incident neutron energy.

The Fission Table tag includes the following attributes:

- GridIndex

Description: grid value corresponding to the incident neutron energy entry in the IncidentNeutronEnergy tag.

Data type: integer

Default value: $\mathrm{N} / \mathrm{A}$

- TotalFissionEnergy

Description: Total recoverable energy in units of $[\mathrm{MeV}]$ for the fission reaction of this isotope and subsequent decay of fission products corresponding to the incident neutron energy entry in the IncidentNeutronEnergy tag.

Data type: real

Default value: N/A

- PromptFissionEnergy

Description: Prompt recoverable energy in $[\mathrm{MeV}]$ for the fission reaction of this isotope corresponding to the incident neutron energy entry in the IncidentNeutronEnergy tag.

Data type: real

Default value: N/A 
- PromptFissionGammaEnergyFraction

Description: Fraction of PromptFissionEnergy released as gamma rays corresponding to the incident neutron energy entry in the IncidentNeutronEnergy tag.

Data type: real

Default value: N/A

The Fission Table tag includes the following tags:

- YieldFractions

Description: list of yield fractions for the various fission products corresponding to the incident neutron energy entry in the IncidentNeutronEnergy tag.

Data type: real vector

Default value: empty vector

- FissionProducts

Description: list of fission products for fission reactions corresponding to the incident neutron energy entry in the IncidentNeutronEnergy tag.

Data type: string vector

Default value: empty vector

The Spontaneous tag includes the following attributes:

- TotalSFEnergy

Description: Total recoverable energy in units of $[\mathrm{MeV}]$ for the spontaneous fission of this isotope and subsequent decay reactions of fission products.

Data type: real

Default value: 0.0

- PromptSFEnergy

Description: Prompt recoverable energy in units of $[\mathrm{MeV}]$ for the spontaneous fission of this isotope.

Data type: real

Default value: 0.0

- PromptSFGammaEnergyFraction

Description: Fraction of prompt recoverable energy released in the form of gamma rays for the spontaneous fission of this isotope.

Data type: real

Default value: 0.0

- BranchingRatio

Description: Branching ratio for spontaneous fission of this isotope.

Data type: real

Default value: 0.0 
The Spontaneous tag includes the following tags:

- YieldFractions

Description: list of yield fractions for the various spontaneous fission products.

Data type: real vector

Default value: empty vector

- SFProducts

Description: list of spontaneous fission products.

Data type: string vector

Default value: empty vector

\subsection{A Sample Valid Library}

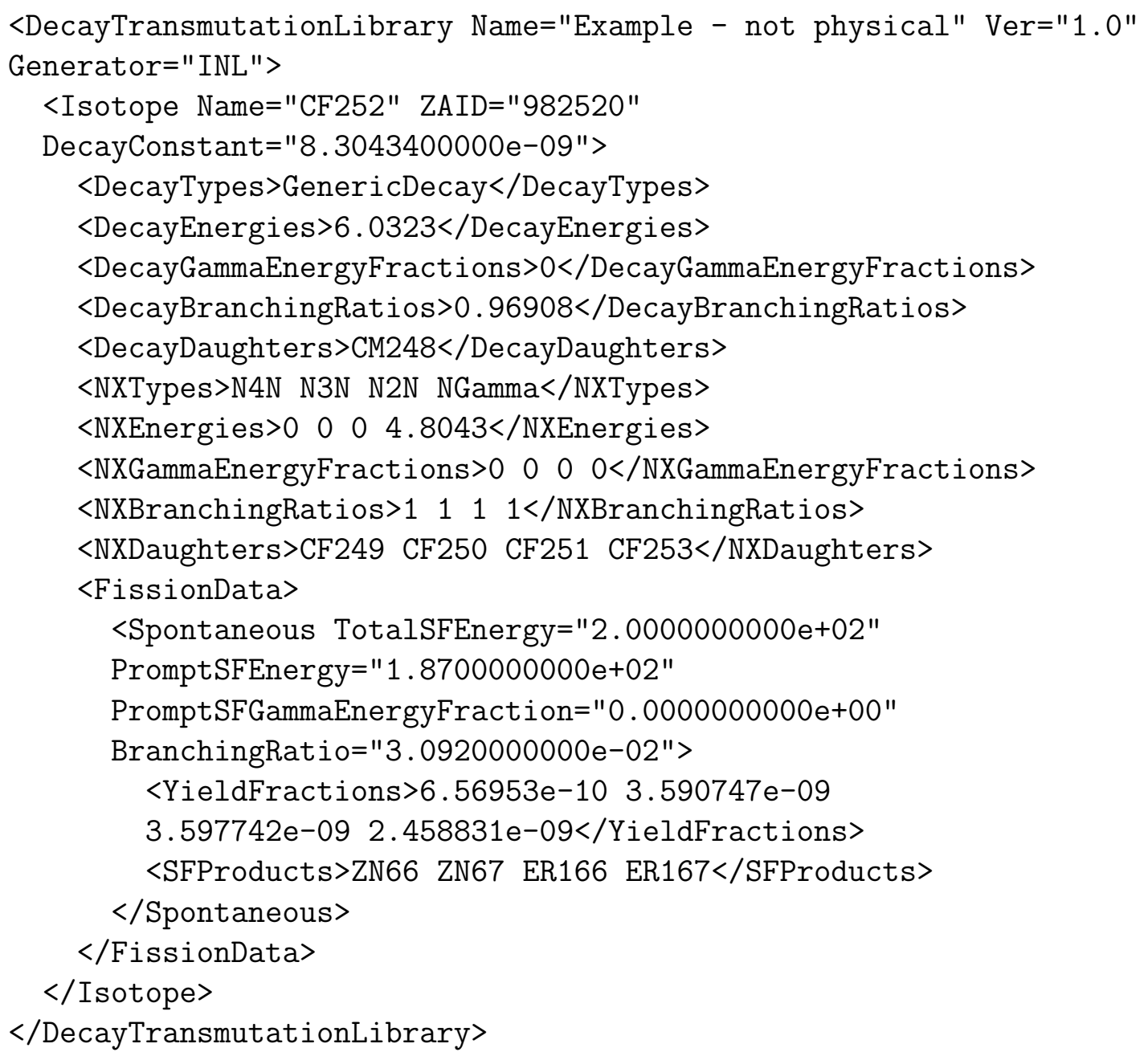

\section{Validation}

In order to validate the depletion implementation in MAMMOTH several test problems were performed, each testing a separate aspect of the depletion implementation. The first problem analyzed is a pure radioactive decay problem involving thorium-232 and its 
decay daughters, resulting in a total of twelve isotopes tracked. This is the simplest type of depletion problem to analyze since cross sections and neutron fluxes are not a factor and the resulting Bateman equations can be solved analytically rather easily. The second problem analyzed is a homogenized light water reactor fuel pin in which 297 isotopes are tracked. The data and basis for comparison come from the DRAGON-5 depletion code. This problem involves a constant neutron flux with one-group cross sections which are updated at each time step to account for burnup while in addition to tracking radioactive decay. Lastly, a sample LWR fuel pellet where 1,450 isotopes are tracked. The data and basis for comparison come from the ORIGEN depletion code. This problem involves a constant power level with one-group cross sections. In order to maintain a constant power level, the neutron flux within the depletion system must change in order to account for changing reaction rates as a result of changes in nuclide concentrations after each depletion time step.

For all problems tested, the data used by MAMMOTH was stored in the ISOXML and, when necessary, ISOXML writers were developed to be able to transfer the data used in the problem into the ISOXML format.

\subsection{Thorium-232 Decay Series}

The thorium-232 decay series (shown in Figure 1 [18]) was selected since it includes one dozen isotopes with half-lives ranging from billions of years to hundreds of nanoseconds. The problem statement is the radioactive decay of one mole $\left(6.023 \times 10^{23}\right.$ atoms $)$ of thorium-232 for one year (no neutron flux is present in the system). A semi-analytical benchmark solution for the number densities of the isotopes in the thorium-232 series is available [19], with the relevant decay information used by the benchmark shown in Tables 3 and 4 while the reference number density solutions for the benchmark are shown in Table 5. The benchmark solution was obtained with the Doubling algorithm where the time steps are found adaptively with Richardson or Wynn-Epsilon extrapolation. These results were confirmed via a Transmutation Trajectory Analysis (TTA) solution.

Table 3: Decay constants from the thorium-232 series benchmark

\begin{tabular}{c|c} 
Isotope & Decay Constant $\left[d^{-1}\right]$ \\
\hline Th-232 & $1.351625 \mathrm{E}-13$ \\
Ra-228 & $3.302667 \mathrm{E}-04$ \\
Ac-228 & $2.661685 \mathrm{E}+00$ \\
Th-228 & $9.934261 \mathrm{E}-04$ \\
Ra-224 & $1.908497 \mathrm{E}-01$ \\
Rn-220 & $1.077121 \mathrm{E}+03$ \\
Po-216 & $4.130201 \mathrm{E}+05$ \\
Pb-212 & $1.563490 \mathrm{E}+00$ \\
Bi-212 & $1.648442 \mathrm{E}+01$ \\
Po-212 & $2.002940 \mathrm{E}+11$ \\
Tl-208 & $3.269348 \mathrm{E}+02$ \\
\hline
\end{tabular}




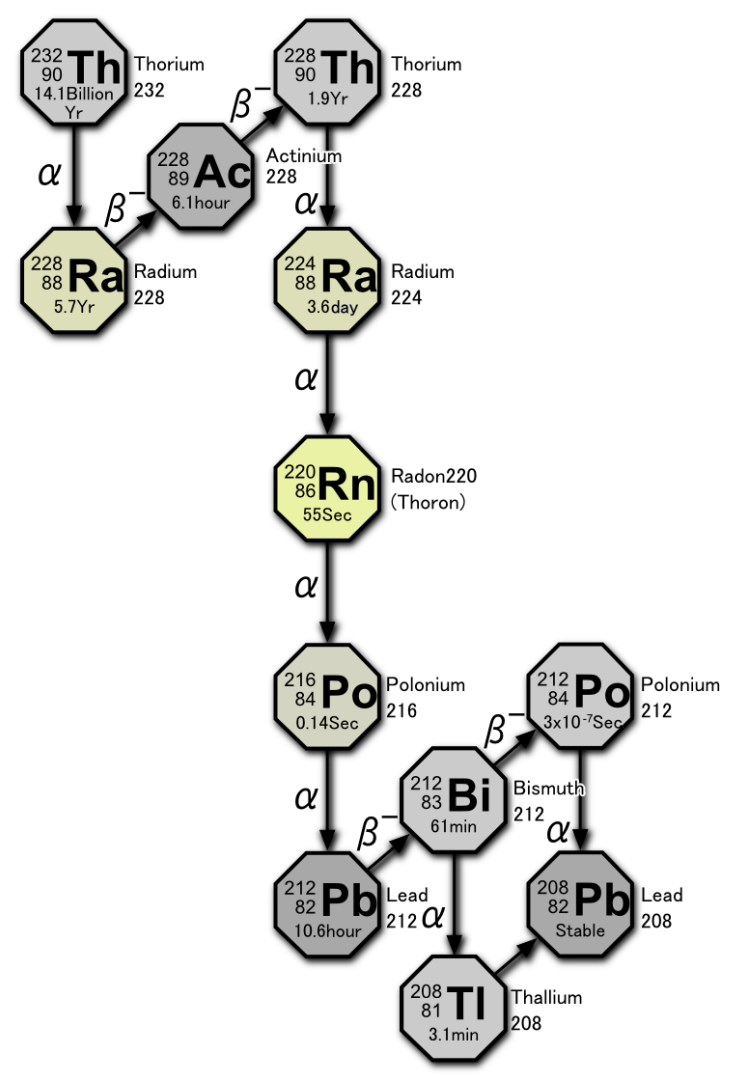

Figure 1: Thorium-232 decay series, image taken from Tosaka [18].

Table 4: Branching ratios for the radioactive decay of Bi-212 from the thorium-232 series benchmark

\begin{tabular}{c|c} 
Daughter Isotope & Branching Ratio \\
\hline Po-212 & 0.6406 \\
Tl-208 & 0.3594 \\
\hline
\end{tabular}

The number density results obtained by MAMMOTH for the thorium-232 decay series are shown in Table 6. The relative error for the concentration of $\mathrm{Pb}-208$ is shown in Table 7. Since error in depletion calculations compounds as the nuclide becomes further and further removed from the original progenitor nuclide, the final nuclide in the system will demonstrate the maximum error present in the system. With precision comparable to that of the reference solution, MAMMOTH matches the number density solution of the benchmark, validating the depletion functionality of MAMMOTH for radioactive decay. It should be noted that the relative error between MAMMOTH and the benchmark solution decreases over time and this is attributed to the increasing concentration of lead-208 as time passes with the increasing concentration of lead able to be calculated more precisely in double precision particularly as more of the intermediate nuclides begin to reach secular equilibrium. 
Table 5: Reference solution number densities for isotopes in the thorium-232 series after 1 year. ( $1 \times 10^{-12}$ relative error)

\begin{tabular}{|c|c|c|c|c|}
\hline Time (days) & Th-232 & Ra-228 & Ac- 228 & Th-228 \\
\hline 36.5 & $6.022999999970 \mathrm{E}+23$ & $2.953567638154 \mathrm{E}+12$ & $3.627339589142 \mathrm{E}+08$ & $1.726783939692 \mathrm{E}+10$ \\
\hline 73.0 & $6.022999999941 \mathrm{E}+23$ & $5.871744543953 \mathrm{E}+12$ & $7.248715742555 \mathrm{E}+08$ & $6.867693005511 \mathrm{E}+10$ \\
\hline 109.5 & $6.022999999911 \mathrm{E}+23$ & $8.754954782151 \mathrm{E}+12$ & $1.082669923668 \mathrm{E}+09$ & $1.525986405848 \mathrm{E}+11$ \\
\hline 146.0 & $6.022999999881 \mathrm{E}+23$ & $1.160361733620 \mathrm{E}+13$ & $1.436181001830 \mathrm{E}+09$ & $2.674636649886 \mathrm{E}+11$ \\
\hline 182.5 & $6.022999999851 \mathrm{E}+23$ & $1.441814616915 \mathrm{E}+13$ & $1.785456180401 \mathrm{E}+09$ & $4.117635120055 \mathrm{E}+11$ \\
\hline 219.0 & $6.022999999822 \mathrm{E}+23$ & $1.719895028378 \mathrm{E}+13$ & $2.130546215485 \mathrm{E}+09$ & $5.840482803810 \mathrm{E}+11$ \\
\hline 255.5 & $6.022999999792 \mathrm{E}+23$ & $1.994643378205 \mathrm{E}+13$ & $2.471501255008 \mathrm{E}+09$ & $7.829245140706 \mathrm{E}+11$ \\
\hline 292.0 & $6.022999999762 \mathrm{E}+23$ & $2.266099592383 \mathrm{E}+13$ & $2.808370846007 \mathrm{E}+09$ & $1.007053134518 \mathrm{E}+12$ \\
\hline 328.5 & $6.022999999733 \mathrm{E}+23$ & $2.534303118491 \mathrm{E}+13$ & $3.141203941827 \mathrm{E}+09$ & $1.255147447262 \mathrm{E}+12$ \\
\hline 365.0 & $6.022999999703 \mathrm{E}+23$ & $2.799292931431 \mathrm{E}+13$ & $3.470048909235 \mathrm{E}+09$ & $1.525971220201 \mathrm{E}+12$ \\
\hline Time (days) & Ra-224 & Rn-220 & Po-216 & $\mathrm{Pb}-212$ \\
\hline 36.5 & $6.770295695164 \mathrm{E}+07$ & $1.199525306804 \mathrm{E}+04$ & $3.128258654688 \mathrm{E}+01$ & $7.939993206442 \mathrm{E}+06$ \\
\hline 73.0 & $3.102328977782 \mathrm{E}+08$ & $5.496713712636 \mathrm{E}+04$ & $1.433495695291 \mathrm{E}+02$ & $3.717037958899 \mathrm{E}+07$ \\
\hline 109.5 & $7.231989443712 \mathrm{E}+08$ & $1.281377826921 \mathrm{E}+05$ & $3.341723329185 \mathrm{E}+02$ & $8.722319214702 \mathrm{E}+07$ \\
\hline 146.0 & $1.298389797172 \mathrm{E}+09$ & $2.300522691959 \mathrm{E}+05$ & $5.999565686358 \mathrm{E}+02$ & $1.570954782267 \mathrm{E}+08$ \\
\hline 182.5 & $2.027908498993 \mathrm{E}+09$ & $3.593113727562 \mathrm{E}+05$ & $9.370532207864 \mathrm{E}+02$ & $2.458226146684 \mathrm{E}+08$ \\
\hline 219.0 & $2.904164707130 \mathrm{E}+09$ & $5.145701970348 \mathrm{E}+05$ & $1.341954915050 \mathrm{E}+03$ & $3.524774300961 \mathrm{E}+08$ \\
\hline 255.5 & $3.919863473831 \mathrm{E}+09$ & $6.945361853018 \mathrm{E}+05$ & $1.811290774316 \mathrm{E}+03$ & $4.761688345707 \mathrm{E}+08$ \\
\hline 292.0 & $5.067994426381 \mathrm{E}+09$ & $8.979672033074 \mathrm{E}+05$ & $2.341821418979 \mathrm{E}+03$ & $6.160404979930 \mathrm{E}+08$ \\
\hline 328.5 & $6.341821336098 \mathrm{E}+09$ & $1.123669691062 \mathrm{E}+06$ & $2.930434146939 \mathrm{E}+03$ & $7.712695760090 \mathrm{E}+08$ \\
\hline 365.0 & $7.734872062336 \mathrm{E}+09$ & $1.370496881064 \mathrm{E}+06$ & $3.574138286889 \mathrm{E}+03$ & $9.410654817207 \mathrm{E}+08$ \\
\hline Time (days) & Bi-212 & Po-212 & Tl-208 & $\mathrm{Pb}-208$ \\
\hline 36.5 & $7.501737681487 \mathrm{E}+05$ & $3.955073325537 \mathrm{E}-05$ & $1.359150693723 \mathrm{E}+04$ & $1.310116157156 \mathrm{E}+08$ \\
\hline 73.0 & $3.519211116439 \mathrm{E}+06$ & $1.855401855481 \mathrm{E}-04$ & $6.376713399337 \mathrm{E}+04$ & $1.313896772178 \mathrm{E}+09$ \\
\hline 109.5 & $8.263344020327 \mathrm{E}+06$ & $4.356608148962 \mathrm{E}-04$ & $1.497343094422 \mathrm{E}+05$ & $4.761869212390 \mathrm{E}+09$ \\
\hline 146.0 & $1.488744026561 \mathrm{E}+07$ & $7.848970515905 \mathrm{E}-04$ & $2.697691038058 \mathrm{E}+05$ & $1.163451205090 \mathrm{E}+10$ \\
\hline 182.5 & $2.330000268061 \mathrm{E}+07$ & $1.228424972983 \mathrm{E}-03$ & $4.222134591463 \mathrm{E}+05$ & $2.303525827430 \mathrm{E}+10$ \\
\hline 219.0 & $3.341308648481 \mathrm{E}+07$ & $1.761607945931 \mathrm{E}-03$ & $6.054736918563 \mathrm{E}+05$ & $4.001356399748 \mathrm{E}+10$ \\
\hline 255.5 & $4.514216930889 \mathrm{E}+07$ & $2.379989773984 \mathrm{E}-03$ & $8.180181372398 \mathrm{E}+05$ & $6.356700654227 \mathrm{E}+10$ \\
\hline 292.0 & $5.840602583819 \mathrm{E}+07$ & $3.079288088324 \mathrm{E}-03$ & $1.058374877876 \mathrm{E}+06$ & $9.464330569818 \mathrm{E}+10$ \\
\hline 328.5 & $7.312660696192 \mathrm{E}+07$ & $3.855387976254 \mathrm{E}-03$ & $1.325129553631 \mathrm{E}+06$ & $1.341422709222 \mathrm{E}+11$ \\
\hline 365.0 & $8.922892326710 \mathrm{E}+07$ & $4.704335838763 \mathrm{E}-03$ & $1.616923250406 \mathrm{E}+06$ & $1.829176771398 \mathrm{E}+11$ \\
\hline
\end{tabular}


Table 6: MAMMOTH solution number densities for isotopes in the thorium-232 series after 1 year.

\begin{tabular}{|c|c|c|c|c|}
\hline Time (days) & Th-232 & Ra-228 & Ac-228 & Th-228 \\
\hline 36.5 & $.022999999970 \mathrm{E}+23$ & $2.953567638154 \mathrm{E}+12$ & $3.627339589142 \mathrm{E}+08$ & $1.726783939691 \mathrm{E}+10$ \\
\hline 73.0 & $.022999999940 \mathrm{E}+23$ & $5.871744543953 \mathrm{E}+12$ & $7.248715742555 \mathrm{E}+08$ & $6.867693005509 \mathrm{E}+10$ \\
\hline 109.5 & $.022999999911 \mathrm{E}+23$ & $8.754954782151 \mathrm{E}+12$ & $1.082669923668 \mathrm{E}+09$ & $1.525986405847 \mathrm{E}+11$ \\
\hline 146.0 & $022999999881 \mathrm{E}+23$ & $1.160361733620 \mathrm{E}+13$ & $1.436181001830 \mathrm{E}+09$ & $2.674636649886 \mathrm{E}+11$ \\
\hline 182.5 & $022999999851 \mathrm{E}+23$ & $1.441814616915 \mathrm{E}+13$ & $1.785456180401 \mathrm{E}+09$ & $4.117635120054 \mathrm{E}+11$ \\
\hline 219.0 & $.022999999821 E+23$ & $1.719895028378 \mathrm{E}+13$ & $2.130546215484 \mathrm{E}+09$ & $5.840482803809 \mathrm{E}+11$ \\
\hline 255.5 & $022999999792 \mathrm{E}+23$ & $1.994643378205 \mathrm{E}+13$ & $2.471501255008 \mathrm{E}+09$ & $7.829245140704 \mathrm{E}+11$ \\
\hline 292.0 & $022999999762 \mathrm{E}+23$ & $2.266099592383 \mathrm{E}+13$ & $2.808370846007 \mathrm{E}+09$ & $1.007053134518 \mathrm{E}+12$ \\
\hline 328.5 & $022999999732 \mathrm{E}+23$ & $2.534303118491 \mathrm{E}+13$ & $3.141203941827 \mathrm{E}+09$ & $1.255147447261 \mathrm{E}+12$ \\
\hline 365.0 & $6.022999999702 \mathrm{E}+23$ & $2.799292931430 \mathrm{E}+13$ & $3.470048909235 \mathrm{E}+09$ & $1.525971220200 \mathrm{E}+12$ \\
\hline Time (day & $\mathrm{Ra}-224$ & Rn-220 & Рo-216 & Pb-212 \\
\hline 36.5 & $770295695165 \mathrm{E}+07$ & $1.199525306803 \mathrm{E}+04$ & $3.128258654684 \mathrm{E}+01$ & $7.939993206433 \mathrm{E}+06$ \\
\hline & $102328977781 \mathrm{E}+08$ & $5.496713712634 \mathrm{E}+04$ & & $3.717037958896 \mathrm{E}+07$ \\
\hline 109.5 & $231989443710 \mathrm{E}+08$ & $1.281377826921 \mathrm{E}+05$ & $3.341723329184 \mathrm{E}+02$ & $8.722319214698 \mathrm{E}+07$ \\
\hline 146.0 & $1.298389797172 \mathrm{E}+09$ & $2.300522691959 \mathrm{E}+05$ & 5.99956568 & $1.570954782267 \mathrm{E}+08$ \\
\hline 182.5 & $2.027908498992 \mathrm{E}+09$ & $3.593113727562 \mathrm{E}+05$ & 9.37053220 & $2.458226146684 \mathrm{E}+08$ \\
\hline 219.0 & $2.904164707129 \mathrm{E}+09$ & $5.145701970347 \mathrm{E}+05$ & $1.341954915050 \mathrm{E}+03$ & $3.524774300960 \mathrm{E}+08$ \\
\hline 255.5 & $3.919863473830 \mathrm{E}+09$ & $361853017 \mathrm{E}+05$ & $1.811290774316 \mathrm{E}+03$ & $4.761688345706 \mathrm{E}+08$ \\
\hline 292.0 & $067994426380 \mathrm{E}+09$ & 8.979672033 & 2.34182141 & $6.160404979929 \mathrm{E}+08$ \\
\hline 328.5 & $341821336097 \mathrm{E}+09$ & $1.123669691062 \mathrm{E}+06$ & 2.930434146 & $7.712695760089 \mathrm{E}+08$ \\
\hline 365.0 & $7.734872062335 \mathrm{E}+09$ & $1.370496881064 \mathrm{E}+06$ & $3.574138286889 \mathrm{E}+03$ & $9.410654817206 \mathrm{E}+08$ \\
\hline IIIC ( ua & Bi-212 & Po-212 & Tl-208 & $\mathrm{Pb}-208$ \\
\hline 36.5 & $7.501737681478 \mathrm{E}+05$ & $3.955073325532 \mathrm{E}-05$ & $1.359150693722 \mathrm{E}+04$ & $1.310116157101 \mathrm{E}+08$ \\
\hline & $.519211116437 \mathrm{E}+06$ & $1.855401855480 \mathrm{E}-04$ & $6.376713399333 \mathrm{E}+04$ & $1.313896772166 \mathrm{E}+09$ \\
\hline 109.5 & $8.263344020324 \mathrm{E}+06$ & $4.356608148960 \mathrm{E}-04$ & $1.497343094422 \mathrm{E}+05$ & $4.761869212371 \mathrm{E}+09$ \\
\hline & $.488744026560 \mathrm{E}+07$ & $7.848970515902 \mathrm{E}-04$ & $2.697691038057 \mathrm{E}+05$ & $1.163451205088 \mathrm{E}+10$ \\
\hline 182.5 & $2.330000268060 \mathrm{E}+07$ & $1.228424972983 \mathrm{E}-03$ & $4.222134591462 \mathrm{E}+05$ & $2.303525827426 \mathrm{E}+10$ \\
\hline 219.0 & $3.341308648480 \mathrm{E}+07$ & $1.761607945931 \mathrm{E}-03$ & $6.054736918562 \mathrm{E}+05$ & $4.001356399743 \mathrm{E}+10$ \\
\hline 255.5 & $4.514216930888 \mathrm{E}+07$ & 2.379989773984E-03 & $8.180181372396 \mathrm{E}+05$ & $6.356700654221 \mathrm{E}+10$ \\
\hline 292.0 & $5.840602583818 \mathrm{E}+07$ & 3.079288088323E-03 & $1.058374877876 \mathrm{E}+06$ & $9.464330569818 \mathrm{E}+10$ \\
\hline & $7.312660696190 \mathrm{E}+07$ & $3.855387976254 \mathrm{E}-03$ & $1.325129553631 \mathrm{E}+06$ & $1.341422709221 \mathrm{E}+11$ \\
\hline 365.0 & $8.922892326708 \mathrm{E}+07$ & $4.704335838762 \mathrm{E}-03$ & $1.616923250406 \mathrm{E}+06$ & $1.829176771397 \mathrm{E}+11$ \\
\hline
\end{tabular}


Table 7: Relative Error between Reference and MAMMOTH solutions for the number density of Pb-208 after 1 year.

\begin{tabular}{c|c} 
Time [Days] & Relative Error \\
\hline 36.5 & $4.198103859300 \mathrm{E}-11$ \\
73 & $9.133201464106 \mathrm{E}-12$ \\
109.5 & $3.990041002454 \mathrm{E}-12$ \\
146 & $1.719062877426 \mathrm{E}-12$ \\
182.5 & $1.736508227996 \mathrm{E}-12$ \\
219 & $1.249652536849 \mathrm{E}-12$ \\
255.5 & $9.438474746149 \mathrm{E}-13$ \\
292 & $0.000000000000 \mathrm{E}+00$ \\
328.5 & $7.454089157664 \mathrm{E}-13$ \\
365 & $5.465605484433 \mathrm{E}-13$ \\
\hline
\end{tabular}

\subsubsection{Comparison to ORIGEN}

In order to further verify the depletion implementation of MAMMOTH, the thorium232 decay series results for both MAMMOTH and ORIGEN (as released with SCALE 6.2) were compared using the ORIGEN decay data; the half-lives used are shown in Table 8. Both were set to use CRAM as their solution method (as ORIGEN in SCALE 6.2 utilizes CRAM by default when performing depletion calculations) with a CRAM approximation order of 16 (which is the default setting in ORIGEN). The results of the MAMMOTH and ORIGEN inputs and the relative difference between the two are shown in Table 9. Since ORIGEN can only display six decimal places of precision, the MAMMOTH results are also only shown to six decimal places of precision. There is a larger relative error between ORIGEN and MAMMOTH, but this is attributed to a lack of precision within ORIGEN since further experimentation with ORIGEN revealed that decaying thorium-232 over a period equal to its half-life did not precisely result in half of the original thorium-232 remaining at the end of the depletion interval, while MAMMOTH calculates the depletion with much greater precision and accuracy.

Table 8: Half-Lives for the thorium-232 series from ORIGEN

\begin{tabular}{c|c} 
Isotope & Half-Life \\
\hline Th-232 & $1.4050 \mathrm{E}+10[\mathrm{y}]$ \\
Ra-228 & $5.7500 \mathrm{E}+00[\mathrm{y}]$ \\
Ac-228 & $6.1500 \mathrm{E}+00[\mathrm{~h}]$ \\
Th-228 & $1.9120 \mathrm{E}+00[\mathrm{y}]$ \\
Ra-224 & $3.6600 \mathrm{E}+00[\mathrm{~d}]$ \\
Rn-220 & $5.5600 \mathrm{E}+01[\mathrm{~s}]$ \\
Po-216 & $1.4500 \mathrm{E}-01[\mathrm{~s}]$ \\
Pb-212 & $1.0640 \mathrm{E}+01[\mathrm{~h}]$ \\
Bi-212 & $1.0092 \mathrm{E}+00[\mathrm{~h}]$ \\
Po-212 & $1.0000 \mathrm{E}-03[\mathrm{~s}]$ \\
Tl-208 & $3.0530 \mathrm{E}+00[\mathrm{~m}]$ \\
\hline
\end{tabular}


Table 9: ORIGEN and MAMMOTH number density solutions for the thorium-232 series after 1 year.

\begin{tabular}{c|c|c|c} 
Isotope & ORIGEN Number Density & MAMMOTH Number Density & Relative Difference \\
\hline Th-232 & $6.023000 \mathrm{E}+06$ & $6.023000 \mathrm{E}+06$ & $0.000000 \mathrm{E}+00$ \\
Ra-228 & $2.799553 \mathrm{E}-04$ & $2.799293 \mathrm{E}-04$ & $9.287197 \mathrm{E}-05$ \\
Ac-228 & $3.412654 \mathrm{E}-08$ & $3.412246 \mathrm{E}-08$ & $1.195550 \mathrm{E}-04$ \\
Th-228 & $1.526333 \mathrm{E}-05$ & $1.526056 \mathrm{E}-05$ & $1.814807 \mathrm{E}-04$ \\
Ra-224 & $7.788272 \mathrm{E}-08$ & $7.786725 \mathrm{E}-08$ & $1.986320 \mathrm{E}-04$ \\
Rn-220 & $1.369370 \mathrm{E}-11$ & $1.369092 \mathrm{E}-11$ & $2.030131 \mathrm{E}-04$ \\
Po-216 & $3.571197 \mathrm{E}-14$ & $3.570474 \mathrm{E}-14$ & $2.024531 \mathrm{E}-04$ \\
Pb-212 & $9.403015 \mathrm{E}-09$ & $9.401027 \mathrm{E}-09$ & $2.114215 \mathrm{E}-04$ \\
Bi-212 & $8.915940 \mathrm{E}-10$ & $8.914056 \mathrm{E}-10$ & $2.113069 \mathrm{E}-04$ \\
Po-212 & $1.572066 \mathrm{E}-16$ & $1.571747 \mathrm{E}-16$ & $2.029177 \mathrm{E}-04$ \\
Tl-208 & $1.615667 \mathrm{E}-11$ & $1.615269 \mathrm{E}-11$ & $2.463379 \mathrm{E}-04$ \\
Pb-208 & $1.828924 \mathrm{E}-06$ & $1.828397 \mathrm{E}-06$ & $2.881476 \mathrm{E}-04$ \\
\hline
\end{tabular}

\subsection{DRAGON-5 Constant Flux Fuel Pin}

A more complicated neutron transmutation problem is solved using data from the DRAGON-5 depletion code, which is developed by the École Polytechnique de Montréal and freely available to the general public [20]. The DRAGON-5 dataset contains 297 nuclides, all of which are present when performing a neutron transmutation of a typical PWR fuel channel. As DRAGON-5 tracks 297 isotopes, the uranium-235 number density is chosen as the most relevant isotope to track and compare since it undergoes a substantial amount of different types of transmutation reactions when subjected to a neutron flux. The results of the uranium-235 number density for the fuel pin depletion is shown in Table 10. The precision of DRAGON-5 is less than that of MAMMOTH so the relative difference is considered within an acceptable margin given the extra precision MAMMOTH maintains allows for values to diverge over multiple time steps. 
Table 10: U-235 number densities for DRAGON-5 and MAMMOTH

\begin{tabular}{c|c|c|c} 
Time [days] & U-235 DRAGON-5 & U-235 MAMMOTH & Relative Difference \\
\hline 0 & $1.05189485 \mathrm{E}-3$ & $1.051894889161 \mathrm{E}-3$ & $3.72290065 \mathrm{E}-08$ \\
0.5 & $1.05087413 \mathrm{E}-3$ & $1.05087433724479 \mathrm{E}-3$ & $1.97211811 \mathrm{E}-07$ \\
1 & $1.04987528 \mathrm{E}-3$ & $1.04987549870373 \mathrm{E}-3$ & $2.08314010 \mathrm{E}-07$ \\
1.5 & $1.04790088 \mathrm{E}-3$ & $1.04790100845809 \mathrm{E}-3$ & $1.22586108 \mathrm{E}-07$ \\
2 & $1.04202679 \mathrm{E}-3$ & $1.04202692009193 \mathrm{E}-3$ & $1.24845092 \mathrm{E}-07$ \\
2.5 & $1.03235792 \mathrm{E}-3$ & $1.03235837878614 \mathrm{E}-3$ & $4.44406083 \mathrm{E}-07$ \\
3 & $1.0134714 \mathrm{E}-3$ & $1.01347203202355 \mathrm{E}-3$ & $6.23622482 \mathrm{E}-07$ \\
3.5 & $9.95261245 \mathrm{E}-4$ & $9.9526180503666 \mathrm{E}-4$ & $5.62703173 \mathrm{E}-07$ \\
4 & $9.6037722 \mathrm{E}-4$ & $9.60378044498087 \mathrm{E}-4$ & $8.58514831 \mathrm{E}-07$ \\
4.5 & $9.1964961 \mathrm{E}-4$ & $9.19650308582941 \mathrm{E}-4$ & $7.59618591 \mathrm{E}-07$ \\
5 & $8.81832093 \mathrm{E}-4$ & $8.81832912393625 \mathrm{E}-4$ & $9.29194607 \mathrm{E}-07$ \\
5.5 & $8.46530136 \mathrm{E}-4$ & $8.46531069484808 \mathrm{E}-4$ & $1.10271893 \mathrm{E}-06$ \\
6 & $8.13431514 \mathrm{E}-4$ & $8.134323463347 \mathrm{E}-4$ & $1.02323882 \mathrm{E}-06$ \\
6.5 & $7.8228669 \mathrm{E}-4$ & $7.82287515625851 \mathrm{E}-4$ & $1.05540061 \mathrm{E}-06$ \\
7 & $7.52893568 \mathrm{E}-4$ & $7.52894360138787 \mathrm{E}-4$ & $1.05212585 \mathrm{E}-06$ \\
7.5 & $7.25084916 \mathrm{E}-4$ & $7.25085630192086 \mathrm{E}-4$ & $9.84977166 \mathrm{E}-07$ \\
8 & $6.98720047 \mathrm{E}-4$ & $6.98720609881041 \mathrm{E}-4$ & $8.05588795 \mathrm{E}-07$ \\
8.5 & $6.73678762 \mathrm{E}-4$ & $6.73679319138389 \mathrm{E}-4$ & $8.27008985 \mathrm{E}-07$ \\
9 & $6.49857393 \mathrm{E}-4$ & $6.49858008711684 \mathrm{E}-4$ & $9.47456612 \mathrm{E}-07$ \\
9.5 & $6.27165777 \mathrm{E}-4$ & $6.27166131214441 \mathrm{E}-4$ & $5.64785985 \mathrm{E}-07$ \\
10 & $6.05523412 \mathrm{E}-4$ & $6.05523802028506 \mathrm{E}-4$ & $6.44117962 \mathrm{E}-07$ \\
10.5 & $5.84859692 \mathrm{E}-4$ & $5.84860044317976 \mathrm{E}-4$ & $6.02397431 \mathrm{E}-07$ \\
11 & $5.65110939 \mathrm{E}-4$ & $5.65111274812957 \mathrm{E}-4$ & $5.94242535 \mathrm{E}-07$ \\
11.5 & $5.46219875 \mathrm{E}-4$ & $5.46220175719599 \mathrm{E}-4$ & $5.50546790 \mathrm{E}-07$ \\
12 & $5.2813458 \mathrm{E}-4$ & $5.28134895056917 \mathrm{E}-4$ & $5.96546655 \mathrm{E}-07$ \\
12.5 & $5.10807615 \mathrm{E}-4$ & $5.10808186247429 \mathrm{E}-4$ & $1.11832207 \mathrm{E}-06$ \\
13 & $4.94196254 \mathrm{E}-4$ & $4.94196863038657 \mathrm{E}-4$ & $1.23238218 \mathrm{E}-06$ \\
13.5 & $4.78260772 \mathrm{E}-4$ & $4.78261277688989 \mathrm{E}-4$ & $1.05734992 \mathrm{E}-06$ \\
14 & $4.6296441 \mathrm{E}-4$ & $4.62964897914704 \mathrm{E}-4$ & $1.05389247 \mathrm{E}-06$ \\
14.5 & $4.48273611 \mathrm{E}-4$ & $4.4827401964816 \mathrm{E}-4$ & $9.11604319 \mathrm{E}-07$ \\
15 & $4.34156856 \mathrm{E}-4$ & $4.34157326405968 \mathrm{E}-4$ & $1.08349312 \mathrm{E}-06$ \\
\hline & & & \\
& & & \\
10 & & &
\end{tabular}

\subsection{ORIGEN Constant Power Fuel Pellet}

The final test performed was a comparison of a sample $5 \%$ enriched uranium fuel pellet depleted at a constant power level of $30 \mathrm{MW} / \mathrm{MTU}$ over a course of 1100 days for a total burnup of $33 \mathrm{GWd} / \mathrm{MTU}$. This depletion was performed using the Westinghouse $17 \times 17$ assembly [21] with 5\% enrichment one-group library distributed with ORIGEN in SCALE 6.1, as documentation for the ORIGEN cross-section libraries distributed with SCALE 6.2 could not be found. During the depletion, 1,451 nuclides were tracked in the system.

The results of select nuclides are shown in Figures 2 to 4 with a maximum observed relative difference of $1.5 \%$, which is attributed to precision differences between the ORIGEN solution to the depletion system, which in SCALE 6.1 relies on a simplified matrix 
exponential solution, when compared to CRAM as implemented in MAMMOTH. The maximum difference is observed in plutonium-239 during the first day of depletion during which time the concentration of plutonium is increasing by over an order of magnitude with each logarithmic time step, this results in small discrepancies in the scalar neutron flux between ORIGEN and MAMMOTH result in substantial differences during the relatively low plutonium-239 concentration time period.

The differences between CRAM and the ORIGEN matrix exponential method have been documented previously [22]. Indeed, as a result of these differences, CRAM has been implemented as a solver option in ORIGEN in versions of SCALE released after 6.1. Other minor differences between ORIGEN and MAMMOTH contributed to the difference, such as ORIGEN tracking the buildup of hydrogen- 1 as a product of $(\mathrm{n}, \mathrm{p})$ reactions, which MAMMOTH neglects. With these differences accounted for, the differences observed between MAMMOTH and ORIGEN are considered acceptable and demonstrate that MAMMOTH is functioning as intended and producing expected results.

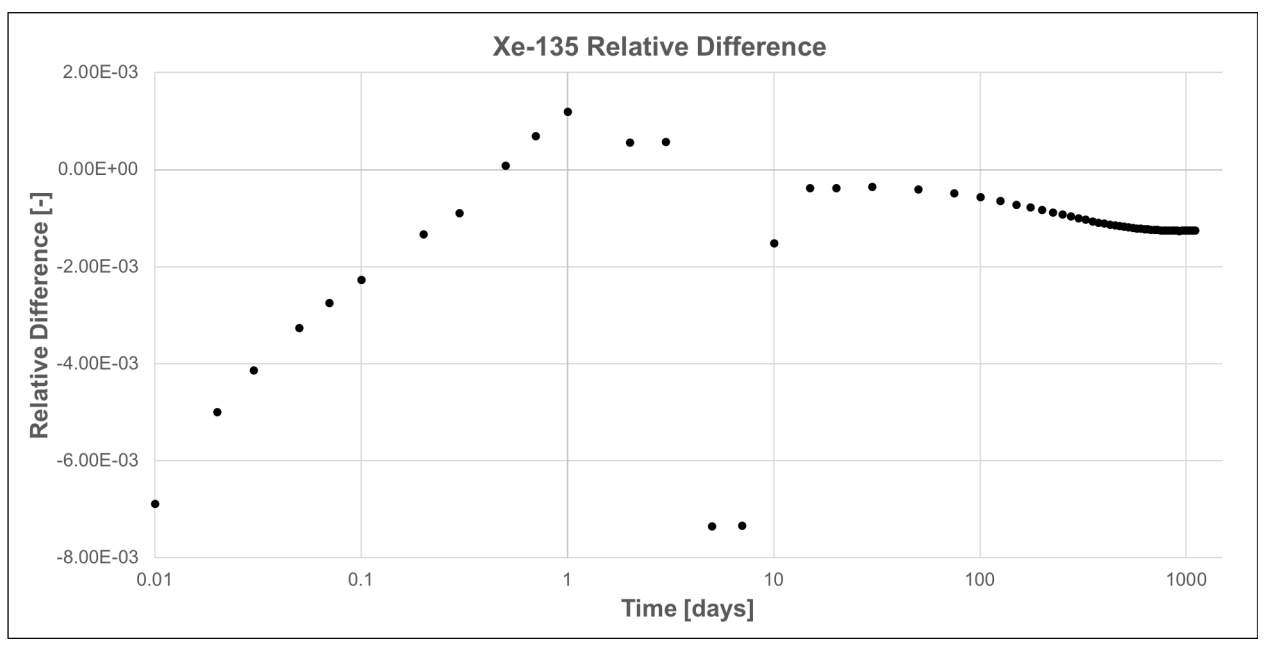

Figure 2: Relative difference between ORIGEN and MAMMOTH for xenon-135.

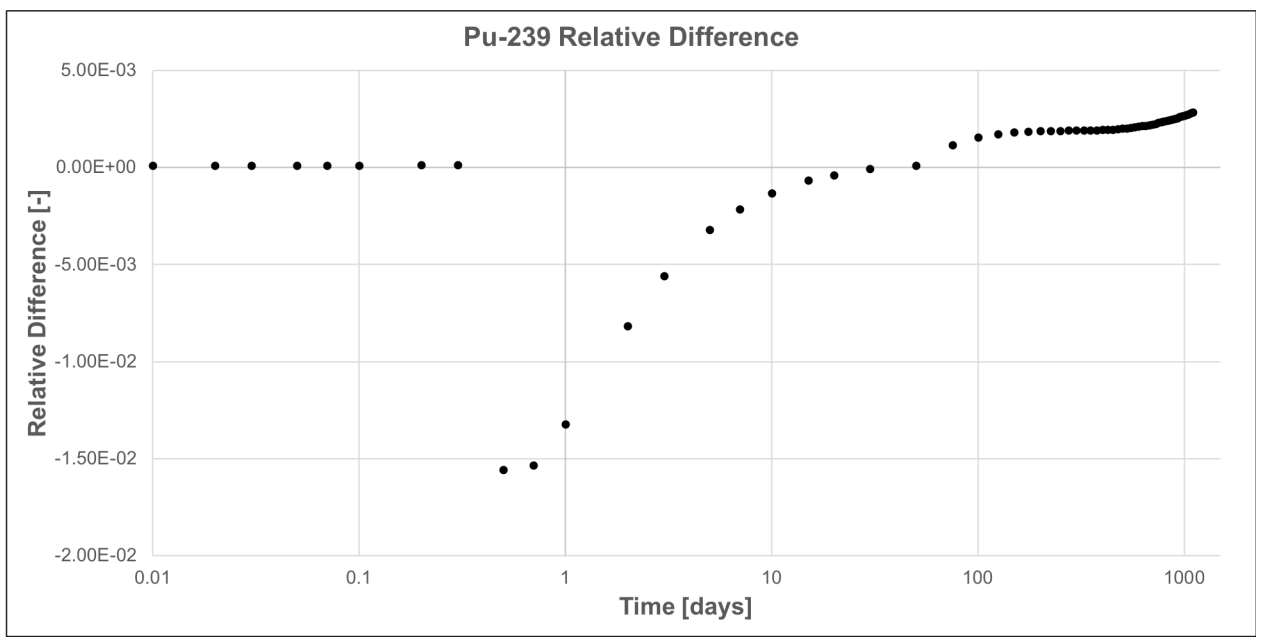

Figure 3: Relative difference between ORIGEN and MAMMOTH for plutonium-239. 


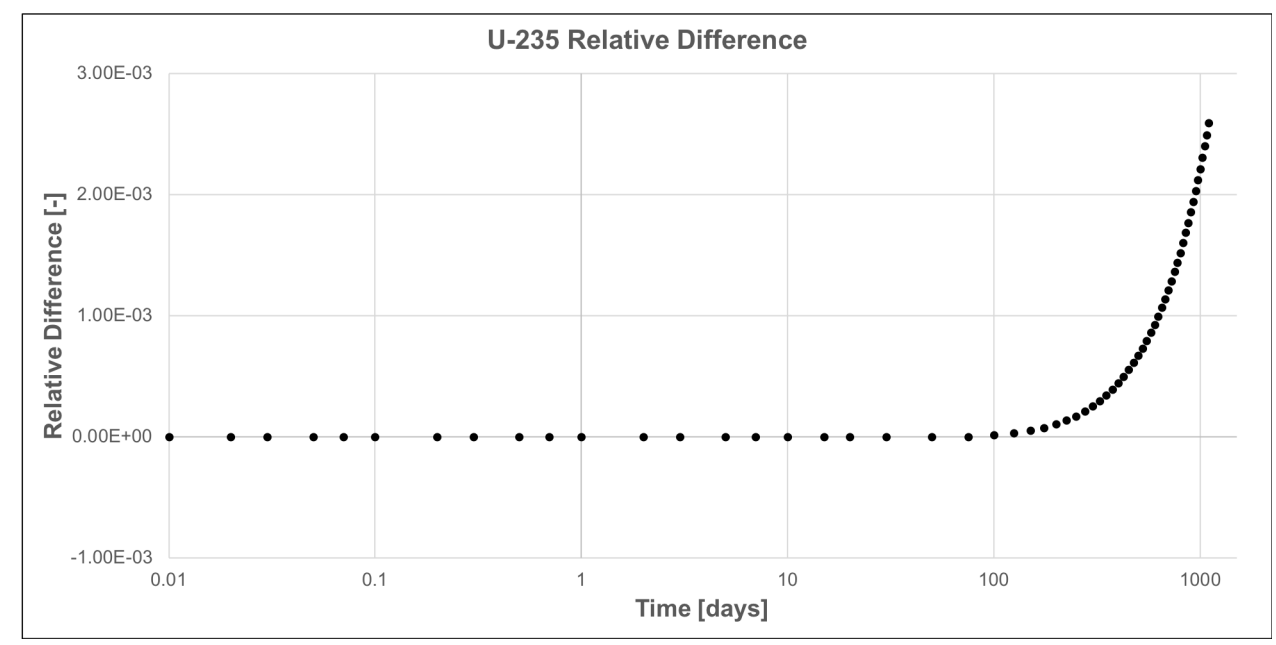

Figure 4: Relative difference between ORIGEN and MAMMOTH for uranium-235.

\section{Conclusion}

The ISOXML format has been developed and added to MAMMOTH allowing the code to store sufficient decay and transmutation data in both human-readable and machinereadable form. The preliminary depletion implementation of MAMMOTH for radioactive decay, constant flux, and constant power problems has been verified against both computational benchmark problems and in code-to-code comparisons. The implementation of MAMMOTH is able to simulate depletion problems involving thousands of nuclides as demonstrated in the ORIGEN fuel pellet comparison.

Future work will include:

- implementation of linear interpolation between neutron energies for the fission product yield fractions,

- addition of the yield fractions for spontaneous fission decay events,

- implementation of multi-group constant-power depletion,

- performance of validation studies against experimental measurements.

\section{Acknowledgements}

The author thanks the Idaho National Laboratory Departments of Reactor Physics Design and Analysis and Nuclear Engineering Methods and Development for their support. The author thanks Drs. Javier Ortensi, Sebastian Schunert, Yaqi Wang, and Sedat Goluoglu, as without their support this work would not have been possible. The author also thanks Dr. Mark DeHart for his role as an adviser and mentor during this project. 


\section{References}

[1] F. N. Gleicher et al., "The Coupling of the Neutron Transport Application Rattlesnake to the Fuels Performance Application BISON," in International Conference on Reactor Physics (PHYSOR 2014), Kyoto, Japan, May 2014.

[2] D. Gaston, C. Newman, G. Hansen, and D. Lebrun-Grandie, "MOOSE: A Parallel Computational Framework for Coupled Systems of Nonlinear Equations," Nuclear Engineering and Design, vol. 239, no. 10, pp. 1768-1778, 2009.

[3] D. R. Gaston, C. J. Permann, J. W. Peterson, A. E. Slaughter, D. Andrs, Y. Wang, M. P. Short, D. M. Perez, M. R. Tonks, J. Ortensi, L. Zou, and R. C. Martineau, "Physics-based multiscale coupling for full core nuclear reactor simulation," Annals of Nuclear Energy, vol. 84, pp. 45-54, 2015. DOI: 10.1016/j . anucene.2014.09. 060.

[4] R.L. Williamson et al., "Multidimensional Multi-physics Simulation of Nuclear Fuel Behavior," Jou. Nucl. Mat., vol. 423, no. 149-163, 2012.

[5] D. Andrs et al., "RELAP-7 Level 2 Milestone Report: Demonstration of a Steady State Single Phase PWR Simulation with RELAP-7," Idaho National Laboratory, Tech. Rep. INL/EXT-12-25924, 2012.

[6] O. W. Hermann and R. M. Westfall, "ORIGEN-S: SCALE system module to calculate fuel depletion, actinide transmutation, fission product buildup and decay, and associated radiation source terms," Vol II, Sect. Fry of SCALE: A Modular Code System for Performing Standardized Computer Analyses for Licensing Evaluation, NUREG/CR-0200, 1998.

[7] H. Bateman, "Solution of a system of differential equations occurring in the theory of radioactive transformations," Proceedings of the Cambridge Philosophical Society, Mathematical and physical sciences., vol. 15, pp. 423-427, 1910.

[8] G. I. Bell and S. Glasstone, Nuclear Reactor Theory. Litton Educational Publishing, Inc., 1970.

[9] H. Moya-Cessa and F. Soto-Eguibar, Differential Equations: An Operational Approach. Rinton Press, 2011.

[10] B. C. Hall, Lie Groups, Lie Algebras, and Representations: An Elementary Introduction. Springer, 2015.

[11] M. Pusa, "Rational approximations to the matrix exponential in burnup calculations," Nuclear Science and Engineering, vol. 169, pp. 155-167, 2011.

[12] Y. Kawamoto, G. Chiba, M. Tsuji, and T. Narabayashi, "Numerical solution of matrix exponential in burn-up equation using mini-max polynomial approximation," Annals of Nuclear Energy, vol. 80, pp. 220-224, 2015.

[13] J. Leppänen, M. Pusa, T. Viitanen, V. Valtavirta, and T. Kaltiaisenaho, "The Serpent Monte Carlo Code: Status, Development and Applications in 2013," Annals of Nuclear Energy, vol. 82, pp. 142-150, 2015.

[14] M. D. DeHart and S. M. Bowman, "Reactor physics methods and analysis capabilities in scale," Nuclear Technology, vol. 174, no. 2, pp. 196-213, 2011. DOI: 10.13182/NT174-196. eprint: https://doi .org/10.13182/NT174-196. [Online]. Available: https://doi .org/10.13182/NT174-196. 
[15] D. I. Poston and H. R. Trellue, "Development of a Fully-Automated Monte Carlo Burnup Code Monteburns," in Transactions of the American Nuclear Society, Jun. 1999.

[16] C. Kang and R. Mosteller, "Incorporation of a predictor-corrector depletion capability into the CELL-2 code," Transactions of the American Nuclear Society, vol. 45, Oct. 1983.

[17] O. W. Hermann and M. D. DeHart, "Validation of SCALE (SAS2H) Isotopic Predictions for BWR Spent Fuel," Oak Ridge National Laboratory, Technical Report ORNL/TM-13315, Sep. 1998.

[18] Tosaka, Decay Chain 4n, Thorium Series, http://www.commons.wikimedia.org, 2010.

[19] K. Huang, Y. Li, and B. Ganapol, "A Backward Euler Doubling Feasibility Study Based on Thorium Series Cascade," in Transactions of the American Nuclear Society, vol. 114, American Nuclear Society, 2016, pp. 399-402.

[20] G. Marleau, A. Hebert, and R. Roy, "A user guide for dragon version 5," Ecole Polytechnique de Montreal, User Manual IGE-355, 2018.

[21] C. E. Sanders and J. C. Wagner, "Study of the Effect of Integral Burnable Absorbers for PWR Burnup Credit," Oak Ridge National Laboratory, Technical Report ORNL/TM-2000/321, 2002.

[22] A. E. Isotalo and P. A. Aarnio, "Comparison of depletion algorithms for large systems of nuclides," Annals of Nuclear Energy, vol. 38, pp. 261-268, 2011. 